\title{
MOLISENS: a modular MObile LIdar SENsor System to exploit the potential of automotive lidar for geoscientific applications
}

\author{
Thomas Goelles ${ }^{2,1^{*}}$, Tobias Hammer ${ }^{1,2,3^{*}}$, Stefan Muckenhuber ${ }^{1,2, *}$, Birgit Schlager ${ }^{2,3, *}$, \\ Jakob Abermann ${ }^{1}$, Christian Bauer ${ }^{1}$, Víctor J. Expósito Jiménez ${ }^{2}$, Wolfgang Schöner ${ }^{1}$, \\ Markus Schratter ${ }^{2}$, Benjamin Schrei ${ }^{1}$, and Kim Senger ${ }^{4}$ \\ ${ }^{1}$ University of Graz - Department of Geography and Regional Sciences, Heinrichstraße 36, 8010 Graz, Austria \\ ${ }^{2}$ Virtual Vehicle Research GmbH, Inffeldgasse 21a, 8010 Graz, Austria \\ ${ }^{3}$ Graz University of Technology - Institute of Automation and Control, Inffeldgasse 21b, 8010 Graz, Austria \\ ${ }^{4}$ University Centre in Svalbard, P.O. Box 156, 9171 Longyearbyen, Norway \\ *These authors contributed equally to this work.
}

Correspondence: thomas.goelles@v2c2.at, hammer.tobias@gmx.de, stefan.muckenhuber@uni-graz.at, birgit.schlager@v2c2.at

\begin{abstract}
We propose a newly developed modular MObile LIdar SENsor System (MOLISENS) to enable new applications for automotive light detection and ranging (lidar) sensors independent of a complete vehicle setup. The stand-alone, modular setup supports both monitoring of dynamic processes and mobile mapping applications based on Simultaneous Localization and Mapping (SLAM) algorithms. The main objective of MOLISENS is to exploit newly emerging perception sensor technologies developed for the automotive industry for geoscientific applications. However, MOLISENS can also be used for other application areas, such as 3D mapping of buildings or vehicle independent data collection for sensor performance assessment and sensor modeling. Compared to Terrestrial Laser Scanners (TLSs), automotive lidar sensors provide advantages in terms of size (in the order of $10 \mathrm{~cm}$ ), weight (in the order of $1 \mathrm{~kg}$ or less), price (typically between 5,000EUR and 10,000EUR), robustness (typical protection class of IP68), frame rates (typically $10 \mathrm{~Hz}-20 \mathrm{~Hz}$ ), and eye safety of class (typically 1). For these reasons, automotive lidar systems can provide a very useful complement to currently used TLS systems that have their strengths in range and accuracy performance. The MOLISENS hardware setup consists of a sensor unit, a data logger, and a battery pack to support stand-alone and mobile applications. The sensor unit includes the automotive lidar Ouster OS1-64 Gen1, a ublox multi-band active Global Navigation Satellite System (GNSS) with the possibility for Real-Time Kinematic (RTK), and a 9axis Xsens Inertial Measurement Unit (IMU). Special emphasis was put on the robustness of the individual components of MOLISENS to support operations in rough field and adverse weather conditions. The sensor unit has a standard screw for easy mounting on various platforms. The current setup of MOLISENS has a horizontal field of view of $360^{\circ}$, a vertical field of view with $45^{\circ}$ opening angle, a range of $120 \mathrm{~m}$, a spatial resolution of a few $\mathrm{cm}$, and a temporal resolution of $10 \mathrm{~Hz}-20 \mathrm{~Hz}$. To evaluate the performance of MOLISENS, we present a comparison between the integrated automotive lidar Ouster OS1-64 and the state of the art TLS RIEGL VZ-6000. The mobile mapping application of MOLISENS has been tested under various conditions and results are shown from two surveys in the Lurgrotte cave system in Austria and a glacier cave in Longyearbreen on Svalbard.
\end{abstract}


Geoscientific Instrumentation Methods and Data Systems

Discussions

\section{Introduction}

Developing new, reliable measurement and monitoring techniques requires emerging cutting-edge technology. This paper introduces a stand-alone, modular MObile SENsor System (MOLISENS) that allows to build on recent lidar, radar and camera innovations, originally developed for high-resolution environment perception of automated vehicles to enable new measurement and monitoring methods for geoscientific applications. MOLISENS includes a Differential Global Positioning System (DGPS) and an Inertial Measurement Unit (IMU) for georeferenced positioning and orientation. The modular setup permits the use of automotive lidar, radar and camera sensors (including traffic monitoring sensors), without the necessity of a complete vehicle setup. This shall allow measuring geoscientific processes reliably, at any remote location, with very high spatial and temporal resolution, and at relatively low costs.

Today, the automotive industry is a leading technology driver for lidar, radar and camera sensors, because the largest challenge for achieving the next level of vehicle automation is to improve the reliability of the vehicle's perception system (Watzenig and Horn, 2017). Both, lidar, radar and camera play essential roles in the perception system of automated vehicles (Marti et al., 2019). The presented work will focus on lidar sensors. However, since MOLISENS is designed as modular system, integration of radar and camera is possible with little effort.

Automotive lidar sensors record high-resolution point clouds with very high acquisition frequencies (around $10 \mathrm{~Hz}-20 \mathrm{~Hz}$ frame rate) to support applications in fast moving environment such as freeways. High costs of mechanically spinning lidars (currently around 5,000EUR to 10,000EUR) are still a limiting factor for many applications, but prices for automotive lidar have already dropped significantly during the last decade and are expected to drop by another order of magnitude in the upcoming years caused by newly emerging technologies like micro-electro-mechanical systems (MEMS) based mirrors, optical phased array, single-photon avalanche diode (SPAD) detectors, and vertical-cavity surface-emitting laser (VCSEL) sources (Hecht, 2018; Druml et al., 2018; Thakur, 2016). One example is the Ouster ES2 (Ouster Inc., 2022), which includes a SPAD detector and VCSEL source. The Ouster ES2 is expected in late 2022 and shall have a range of $200 \mathrm{~m}$ at a cost of around 500EUR. Examples for state-of-the-art automotive lidar types are Ouster OS0 (Ouster Inc., 2021a), Ouster OS1 (Ouster Inc., 2021b), Ouster OS2 (Ouster Inc., 2021c), Velodyne Alpha Prime (Velodyne Lidar Inc., 2021), and Ibeo Lux 4L/8L/HD (Ibeo Automotive Systems GmbH, 2021). In addition to range information, several new lidar types, e.g. Ouster OS (Ouster Inc., 2021a, b, c), provide intensity information for each received point, which allows to take also the reflectance of the illuminated materials for new applications into account.

Due to the above listed advantages, automotive lidar sensors can complement non-automotive Terrestrial Laser Scanner 50 (TLS) systems that are nowadays used for geoscientific applications. Examples for state-of-the-art TLS used in geosciences are Leica P30/P40 (Leica Geosystems AG, 2021a), Leica P50 (Leica Geosystems AG, 2021b), or the Riegl VZ-Series (Riegl Laser Measurement Systems GmbH, 2020). High-end TLS typically provide very detailed and highly accurate point clouds but are more expensive (order of 100,000EUR), heavier (order of $5 \mathrm{~kg}$ to $10 \mathrm{~kg}$ ), less robust (typically IP64) and in certain field scenarios more difficult to handle than small and light-weight automotive lidar sensors. 
Geoscientific Instrumentation Methods and Data Systems

Discussions Laser Measurement Systems GmbH, 2020) was used for ground-truth acquisition. A test setup was designed to compare the accuracy of the VZ-6000 to automotive lidar sensors (Hammer, 2021). The MOLISENS system made it possible to record data with an automotive lidar independent of the vehicle platform.

To support mobile mapping applications, MOLISENS includes a DGPS and an IMU for georeferenced positioning and orientation. For registration of subsequently recorded point clouds into a cumulative point cloud, i.e. for creating a 3D map, the Simultaneous Localization and Mapping (SLAM) algorithm (Bălașa et al., 2021; Zhang and Singh, 2017) LIO-SAM (Shan et al., 2020) is used. Lidar based mobile mapping systems have already been tested in various disciplines such as indoor mapping applications (Tucci et al., 2018), urban mapping applications (Moosmann and Stiller, 2011; Zhang and Singh, 2017; Behley and Stachniss, 2018), and for geoscientific surveys (Bosse et al., 2012; Kukko et al., 2012; Wang et al., 2013). A major advantage of MOLISENS compared to previous systems is the modular setup focused on automotive sensors, that allows to easily exchange and update existing components and extend the system with additional automotive sensors, such as radar and camera.

Apart from newly emerging perception sensor technology, MOLISENS also benefit from recent developments in the Global Navigation Satellite System (GNSS) sector. Typically, DGPS technology for positioning requires extensive additional gear that must be transported into the field, i.e. rover and base station. New GNSS platforms integrate multi-band GNSS and Real-Time Kinematic (RTK) technology to yield accuracies in the order of centimeters with a single device and internet connection. Such GNSS platforms are designed primarily for industrial tracking and wearable applications so they are optimized in size, weight, update rate, and power consumption (Janos and Przemysław, 2021).

The MOLISENS system has been tested under various conditions and results are shown from two mapping surveys in the Lurgrotte cave system in Austria and a glacier cave in Longyearbreen on Svalbard.

This article is organized as follows: Section 2 gives an overview on the hard- and software components of MOLISENS. In Section 3, the integrated automotive lidar OS1-64 is described and compared to the state-of-the-art TLS VZ-6000. In Section 4, the point cloud processing package pointcloudset and the used mapping algorithm are described. The results of two mapping campaigns are shown in Section 5. The discussion of the measurement campaigns and an outlook on future applications are given in Section 6. The conclusion is presented in Section 7.

\section{MOLISENS setup}

MOLISENS provides a stand-alone, modular framework which is capable of integrating various automotive lidar, radar, and camera sensors, that support Robot Operating System (ROS) functionality, with low adjustment effort. The hardware setup follows International Protection (IP) standards of automotive sensors, e.g., the OS1-64 has an IP class of 69K with the cable attached (Ouster Inc., 2020b), which makes it suitable for fieldwork in rough environments. Figure 1 depicts the hardware components of MOLISENS. The data logger and the sensor unit are connected via a self-developed wire harness which avoids the need of multiple cables. The setup can either be powered by batteries or by a Alternating Current (AC)/Direct Current (DC) 
Table 1. Weight and dimensions of hardware setup.

\begin{tabular}{|l|l|l|}
\hline unit & weight in $\mathbf{~ k g}$ & dimensions in cm \\
\hline data logger & 2.1 & $21.5 \cdot 16 \cdot 14$ \\
\hline sensor unit & 1.0 & $10.5 \cdot 10.5 \cdot 15$ \\
\hline wire harness between data logger and sensor unit & 0.7 & 200 \\
\hline Lithium-Ion (Li-ion) battery & 1.7 & $16.7 \cdot 10.0 \cdot 7.9$ \\
\hline total weight & 5.5 & \\
\hline
\end{tabular}

mains adapter. The environment is scanned by the sensor unit and the transmitted sensor data are recorded by the data logger. The data can be downloaded via a Local Area Network (LAN) interface for further post-processing on a computer. The weight of the whole setup, i.e., sensor unit, data logger, battery pack, and wire harness, is $5.438 \mathrm{~kg}$ for mobile measurements (Table 1 ), which require external batteries. Table 1 shows also the dimensions of the modules of MOLISENS.

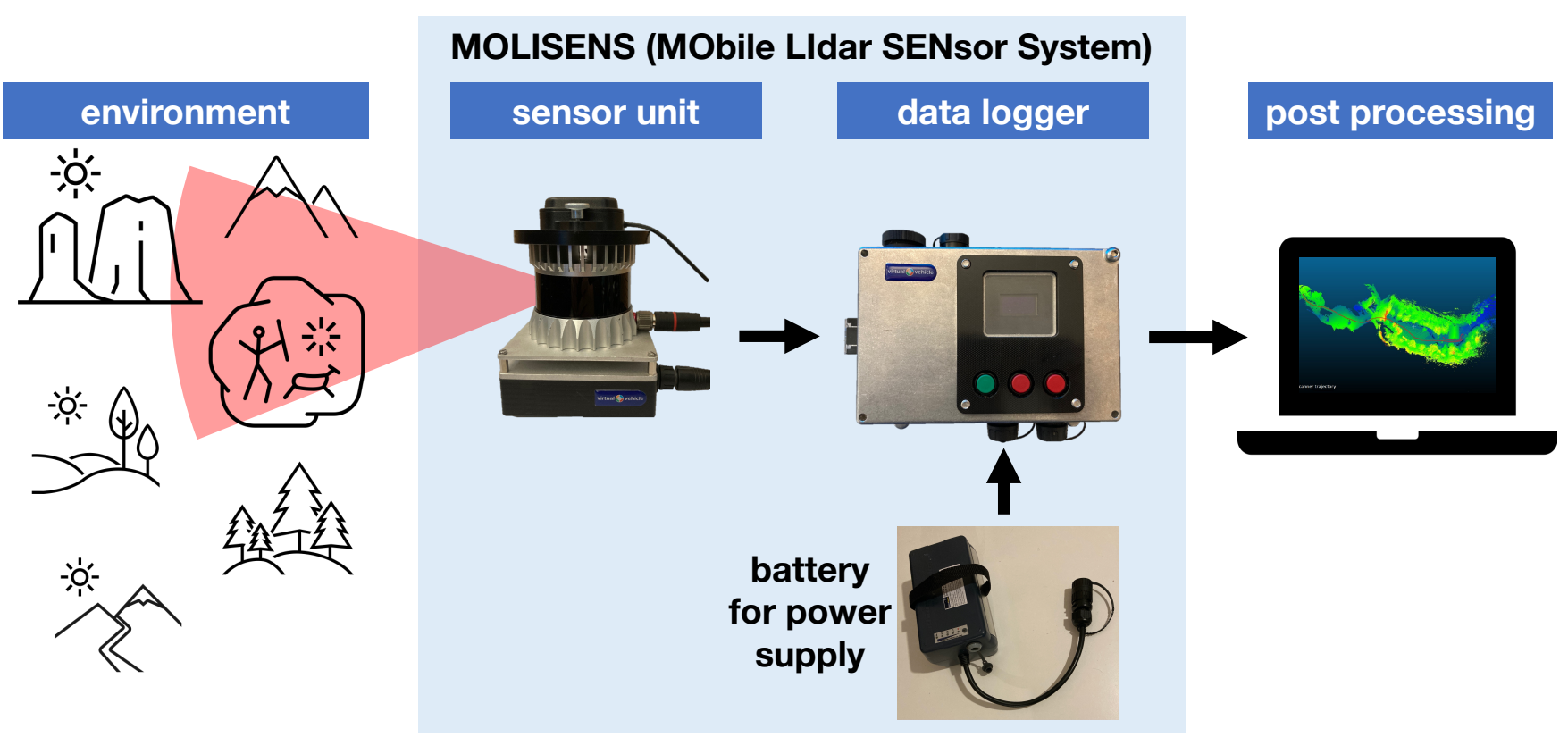

Figure 1. Hardware components and interfaces of MOLISENS.

\subsection{Sensor unit}

The sensor unit consists of the OS1-64 Gen1 which is an automotive rotating lidar sensor, a ublox active multi-band GNSS antenna of the ANN-MB series, and the 9-axis Xsens MTi 630 IMU. Between the OS1-64 and the IMU is a space that heat produced by the $0 S 1-64$ can be dissipated. The sensor unit has a $0.635 \mathrm{~cm}(0.25 \mathrm{inch})$ thread, which is a standard camera thread, mountable on handles, tripods, or other standardized setups. 


\subsection{Data logger}

\subsubsection{Hardware}

The data logger consists of two DC/DC converters, one $24 \mathrm{~V} / 24 \mathrm{~V}$ converter and one $24 \mathrm{~V} / 5 \mathrm{~V}$ converter for internal power supply, a RaspberryPi 4 as processing unit, a RaspberryPi hat for the real-time clock, a RaspberryPi hat with a 1TB Solid State Drive (SSD) for data storage, a RaspberryPi hat for Global Positioning System (GPS) data, a Long Term Evolution (LTE) stick to retrieve RTK data and the Ouster interface board that is responsible for powering the OS1-64 and for data transmission. Interfaces provided by the data logger are a connector for power supply of the whole setup, a 24-pin connector for Ouster data and power supply, the IMU Universal Serial Bus (USB) interface, a Sub-Miniature A (SMA) connector for the GPS antenna, a Registered Jack 45 (RJ45) connector for Ethernet, and a USB connector. Furthermore, the data logger's Human Machine Interface (HMI) includes one on/off button, two red buttons for selecting the measurement programme, one green button for start and stop measurements, and an Organic Light Emitting Diode (OLED) display, which shows the measurement programs, the state of the LTE connection, and the filename of the current measurement. The aluminum housing of the data logger includes an aluminum plate to the integrated circuits on the RaspberryPi which allows for appropriate cooling of the hardware. The OLED display can be seen through a transparent plastic window in the aluminum housing.

\subsubsection{Software}

The software stack of the data logger is shown in Figure 2. Although the official operating system for RaspberryPi is Raspbian (https://www.raspbian.org/), Ubuntu Server 20.04 Long Term Support (LTS) (https://ubuntu.com/download/raspberry-pi) was installed as operating system for MOLISENS, since the integration with ROS (https://www.ros.org) works best on Ubuntu. ROS is an open-source middleware widely used for robotic applications. A major advantage of ROS is the extensive list of opensource third-party packages and tools for different domains, e.g., Autoware (https://www.autoware.org/) for the automotive domain.

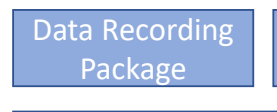

Middleware

\section{Operating System}

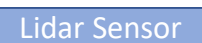
ROS Driver
Robot Operating System (ROS)
GPS Sensor ROS Driver

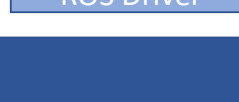


Geoscientific Instrumentation Methods and Data Systems

Discussions

Topics are communication channels, which are defined by a unique name and a specified type of message that is transported. ROS officially supports $\mathrm{C}++$, Python, and Lisp but other programming languages are also possible through unofficial channels.

The specific software packages used in MOLISENS are:

- Data recording package: We developed a ROS package in Python that provides an easy interface to start and stop the data recording as well as a flexible configuration for the specific requirements of the use case. Both, the sampling rate of either $10 \mathrm{~Hz}$ or $20 \mathrm{~Hz}$ of the OS1-64 and the number of points in horizontal direction of either 1,024 or 2,048, can be selected by the user just before the measurements by using the red and green buttons on the data logger. The IMU data are recorded with $200 \mathrm{~Hz}$ and the GPS data with $1 \mathrm{~Hz}$. The ROS package records all messages from the specified topics and creates a time synchronous rosbag file including the data from all sensors. Later, this rosbag file can be used as input to a SLAM algorithm to generate a 3D map of the measurement area.

- Lidar sensor package: The ROS package provided by the sensor manufacturing company was implemented (Ouster Inc., 2020a). It provides transforming the raw data from the sensor into point cloud messages and also includes visualization tools to proof that the lidar sensor is correctly mapping the scenario and to check the light intensity of the points. Due to the computational limitations of the RaspberryPi, only raw data are recorded. The recorded raw data are converted into point cloud messages in a post-processing step.

- IMU sensor package: Similar to the lidar sensor, the ROS driver provided by the manufacturer is used (Xsens, 2021). Only the configuration and topic selection was adopted to meet the requirements of our use case. Most of the topics were omitted to increase the performance of the system.

- GPS sensor package: Another self-developed Python package was used to retrieve the National Marine Electronics Association (NMEA) messages from the ublox GPS module. This driver is also able to receive correction data from Radio Technical Commission for Maritime (RTCM) messages through the integrated Networked Transport of RTCM via Internet Protocol (NTRIP) client. By using this NTRIP client, correction data from the external services are included into the GPS module to improve the accuracy of the measurements. The usage of this correction data is called GPS RTK. The precision is below $2.5 \mathrm{~cm}$ with fixed RTK when the signal from the satellites is clear and also the base station from the correction data service is not far away, i.e., less than 10km, from the GPS module (Dunning, 2018). When the situation is not optimal, the module is still able to reach a precision between $10 \mathrm{~cm}$ and $45 \mathrm{~cm}$ with RTK float (Dunning, 2018).

\subsection{Power supply}

MOLISENS can be powered either with batteries (e.g., Lithium Iron Phosphate (LiFePO4), Li-ion) or with an AC/DC mains adapter that provides a nominal voltage of $24 \mathrm{~V}$. The battery supply supports mobile measurements whereas the mains adapter may be used when recorded data are transferred to the post-processing computer. The described setup including the data logger and the sensor unit draws a current of about $1 \mathrm{~A}$ when data of all three sensors are recorded. We used either a Li-ion-battery with 
Geoscientific Instrumentation Methods and Data Systems

Discussions

10.4Ah for $10.4 \mathrm{~h}$ of measuring or two parallel LiFePO4-batteries with $3.6 \mathrm{Ah}$ each, so $7.2 \mathrm{Ah}$ in total, for $7.2 \mathrm{~h}$ of measuring. The operating temperature for discharging is limited between $-20^{\circ} \mathrm{C}$ to $+60^{\circ} \mathrm{C}$ for both types of batteries. We used batteries from AccuPower (AccuPower Research, Development and Distribution Company (Ltd.), 2022).

\section{MOLISENS with automotive lidar OS1-64 Gen1}

\subsection{OS1-64 Gen1 specifications}

The Ouster OS1-64 is a mechanical spinning lidar scanner that costs about 10,000EUR. The ingress protection level is IP69K with Input/Output (I/O) cable attached, so it offers complete protection against contact, i.e., it is dust-tight and waterproof. It is classified as a mid-range lidar sensor and can detect objects up to a distance of $150 \mathrm{~m}$. The minimum range is $0.8 \mathrm{~m}$. The laser operates with eye safety class 1 per IEC 60825-1:2014, which makes it possible to operate the lidar without any restrictions regarding the eye safety of the operator or other persons within the measurement range. The wavelength of the laser is $855 \mathrm{~nm}$. The range resolution is $0.3 \mathrm{~cm}$, so it is able detect individual objects when the distance between those objects in scanning direction is $0.3 \mathrm{~cm}$ or greater. The range accuracy is stated with $+/-5 \mathrm{~cm}$ for Lambertian targets and $+/-10 \mathrm{~cm}$ for retroreflectors (Ouster Inc., 2020b). The precision depends on the range and is between $+-1 \mathrm{~cm}$ and $+-5 \mathrm{~cm}$. The vertical resolution of the OS1-64 is given by the 64 channels which are evenly distributed within the $33.2^{\circ}$ vertical field of view. The horizontal resolution is configurable and can be 512,1,024, or 2,048 scanning points in horizontal direction for the $360^{\circ}$ field of view. The angular sampling accuracy vertically and horizontally is $+/-0.01^{\circ}$. The sampling frequency can be configured with $10 \mathrm{~Hz}$ or $20 \mathrm{~Hz}$. At $10 \mathrm{~Hz}$, the scanner rotates and scans 10 times per second and produces up to $64 \cdot 2,048$ points per rotation. Therefore, the OS1-64 is able to detect over 1.3 million points per second at $10 \mathrm{~Hz}$ rotation rate.

A computer with ROS installed can read and record the data which are forwarded by the Ouster interface box. In our case, the necessary components are integrated in MOLISENS. We decided to record only raw lidar data to be able to store about 1.3 million points per second, IMU measurements with $200 \mathrm{~Hz}$, and GPS measurements with $1 \mathrm{~Hz}$. The raw lidar data do not comprise the actual 3D-points with $\mathrm{x}-, \mathrm{y}-$, and z-coordinates, but information such as timestamp, measurement id, and range for each measurement. These data is used in a post-processing step for the derivation of 3D-points. Recording the raw data instead of the point cloud data reduces the total data rate, i.e., lidar, IMU, and GPS data, from $77 \mathrm{MB} / \mathrm{s}$ to $15.14 \mathrm{MB} / \mathrm{s}$, a factor of more than 5 . Up to $18 \mathrm{~h}$ of recording are possible with $1 \mathrm{~TB}$ of data storage that we use in MOLISENS.

\subsection{OS1-64 performance assessment}

We assessed the performance of the OS1-64 against the VZ-6000 in a standardized test setup which is based on Boehler et al. (2003). The first frame of each OS1-64 measurement was used for the test. Specifically, the following attributes were analyzed: systematic and surface-induced range errors and angular errors.

We tested the systematic noise in the range measurements of the lidar. For that purpose, a plane target was scanned. We took a wall perpendicular to the observation direction as the plane target. The wall was modeled as a plane. Therefore, the normal 
distance between every point in the point cloud and this plane can be calculated. The standard deviation of the distribution of these normal distances, which represent the range errors, was derived (Figure 3). We investigated two different materials: retroreflective foil and a cardboard with black dull spray paint. Those surfaces need to be in the same plane perpendicular to the observation direction to quantify the deviations of surfaces with high and low reflectivity. This was realized by attaching the materials to a wooden board which was mounted on the wall. We analyzed the reflectance of the materials based on the comparison to Lambertian targets. From a perpendicular angle the reflectivity of the retroreflector was $200 \%$ relative to a $100 \%$ Lambertian target. The black dull spray paint had a reflectivity of $10 \%$ relative to a $100 \%$ Lambertian target. These reflectance values represent the ratio of the measured reflectance to the reflectance of a standard 100\% Lambertian material (Muckenhuber et al., 2020, Birkebak et al., 2018). The scanned wall in the background was used to model a reference plane. The normal distances between points and reference plane can be calculated by subtracting the thickness of the wooden board and the target thickness. A reflectance threshold was used to select the points representing the target.

We used scanned circles to determine the vertical and horizontal distances for quantifying the angular accuracy. We attached four circles, representing a rectangle, with dimensions of $4.5 \mathrm{~m} \cdot 2 \mathrm{~m}$, to a wall. This rectangle was scanned from three different positions which yields six independent vertical and six independent horizontal distances.

The results of the tests on systematic range errors showed that the OS1-64 has a higher standard deviation in the range error distribution compared to a TLS such as the VZ-6000 (Figure 3 (a)). The most significant range errors of up to $25 \mathrm{~cm}$ occurred when scanning retroreflective targets with the OS1-64 (Figure 3d). Furthermore, the range errors in this case are not only larger but are also spread out over a large range of values with a standard deviation of $6.9 \mathrm{~cm}$. According to the manufacturer, the errors occurring with retroreflectors result from the time walk error. This error is caused by clock errors and is an internal error source of lidar systems. This means that the light returns so strongly that it deforms the shape of the received signal which leads to an error in the estimation of the peak, i.e., the distance measured (Nahler et al., 2020). 


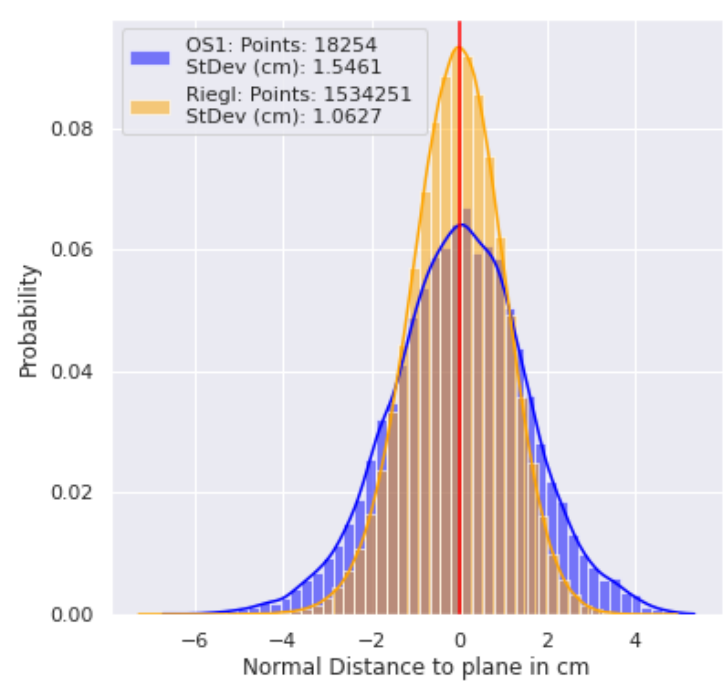

(a)

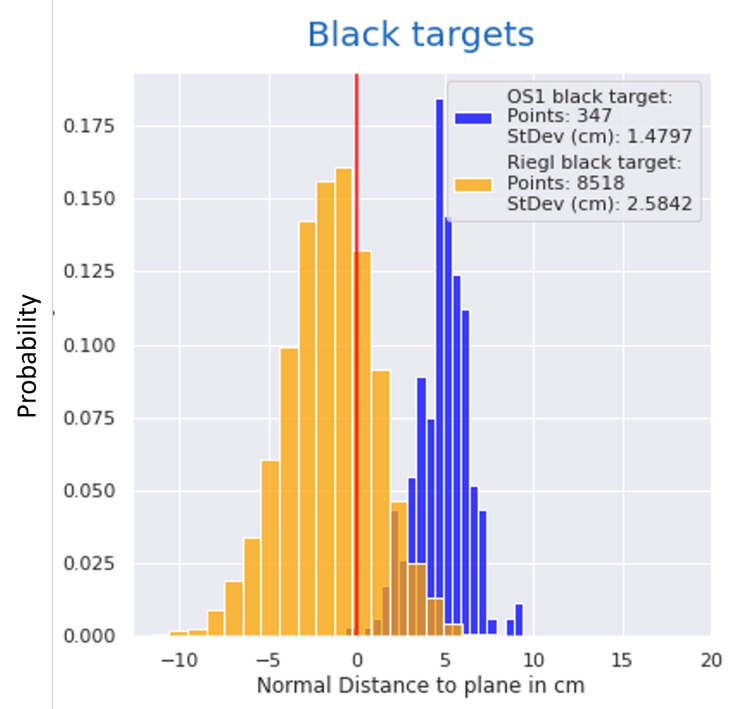

(c)

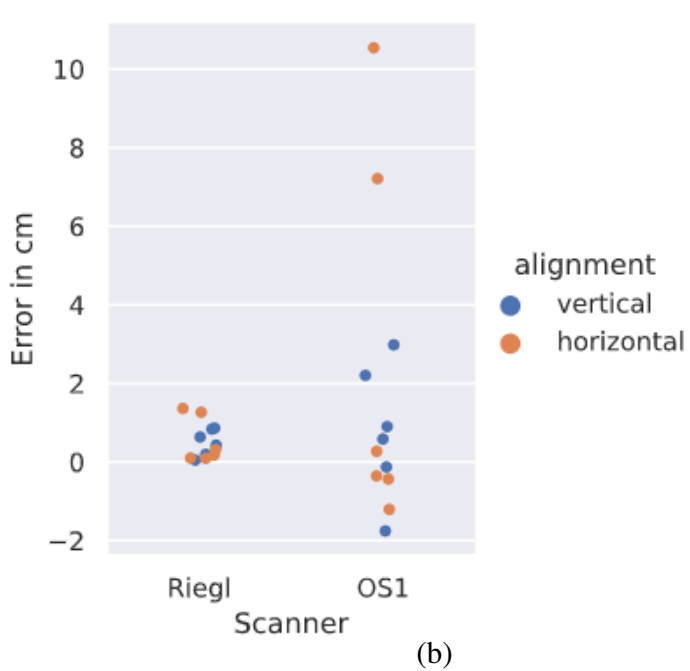

Retroreflector

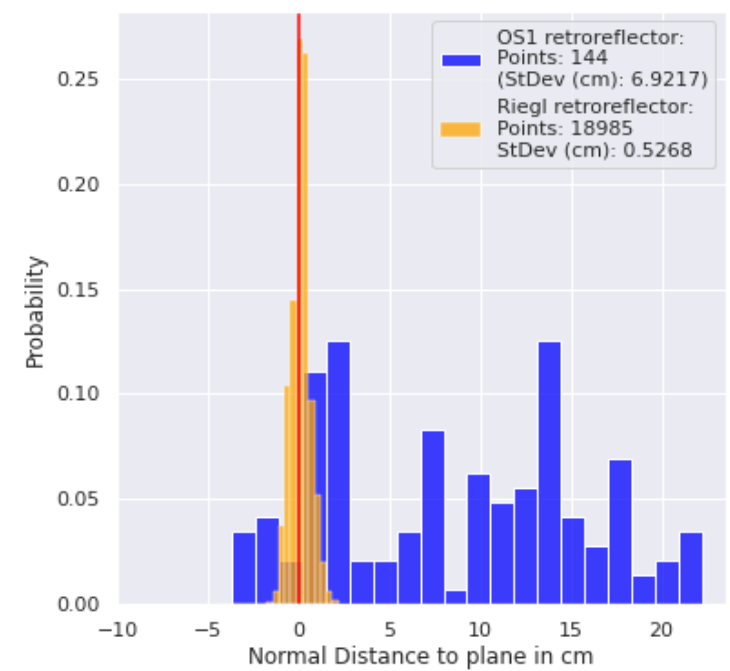

(d)

Figure 3. Results of the accuracy assessment of the OS1-64 compared to the VZ-6000 which was used as the reference lidar: (a) range error for a white wall, i.e. normal distances between measurements and ideal plane of the wall, (b) angular error, (c) range errors for black target with low reflectivity, i.e. normal distances between measurements and ideal plane of black target, (d) range errors for retroreflectors, i.e. normal distances between measurements and ideal plane of retroreflector.

Figure 4 shows parts of point clouds with observed artifacts. Areas without data appear near highly reflecting objects (Figure 4 (a) and (b)). The results also show that the OS1-64 performed better at detecting low reflectance targets in close proximity 
than the VZ-6000 (Figure 4 (c) and (d)). We found that the VZ-6000 shows larger errors when scans are conducted in indoor environments and at low ranges between 5 and $15 \mathrm{~m}$.

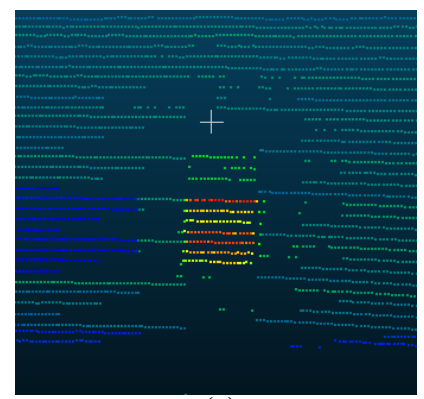

(a)

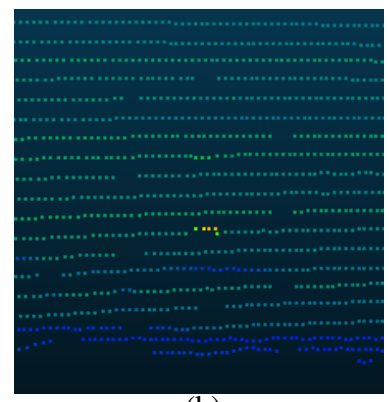

(b)

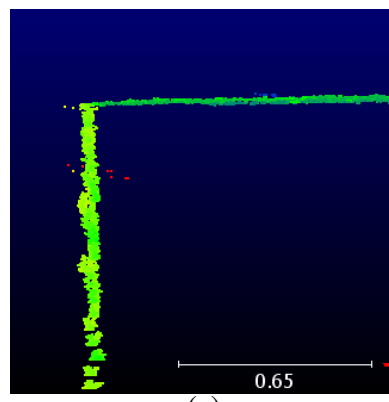

(c)

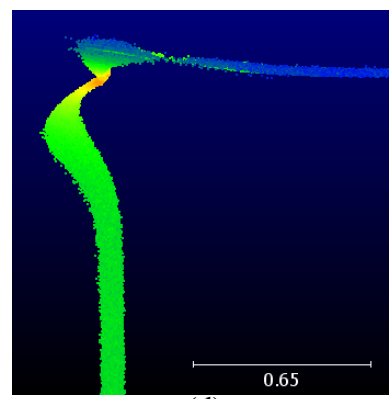

(d)

Figure 4. Artifacts observed in the accuracy tests: (a) and (b) areas without data resulting from blooming filter in the OS1-64 data, (c) a part of the OS1-64 point cloud with a corner of the room, (d) a part of the VZ-6000 point cloud with a corner of the room.

The found performance issues comprise the following artifacts: range errors at highly reflective targets for the OS1-64, areas without data around highly reflective targets for the OS1-64, corner artifacts for the VZ-6000, and multipath artifacts for the VZ-6000. The systematic range errors, which can be considered as noise, can have great impact on mapping microscale features. According to the manufacturer, the effect of areas without data is caused by an integrated blooming filter in the OS1-64. This filter removes bloomed, i.e. overly saturated, points. The range errors of the OS1-64 have a big impact on current point cloud georeferencing methods where retroreflectors of known position are used as distinguishable objects in a point cloud. Other georeferencing methods, like the matching with georeferenced point clouds, must be considered.

\section{Data processing for lidar applications}

\subsection{Data processing of point cloud time series}

Automotive lidar sensors record point clouds in the order of a million points per second at acquisition rates ranging from $10 \mathrm{~Hz}$ to $20 \mathrm{~Hz}$. In addition to the $x-, y$-, and $z$-coordinates, also other parameters such as intensity, reflectivity, noise, etc. are stored for each point. This leads to datasets in the order of gigabytes in the form of 3D point clouds recorded over time. Most software deals with single point clouds and the tools provided by ROS are not designed for post-processing and data analytics. Therefore, a Python package called pointcloudset (Goelles et al., 2021) was developed along the sensor hardware. The package is available on the Python Package Index (PyPI) for easy installation with pip. This package organizes the data in the following way: The point cloud data stored in a ROS bag-file (.bag) is read into a pointcloudset Dataset. This Dataset object consists of multiple PointCloud objects, timestamps, and metadata.

The package is optimized for analytics on the whole dataset to answer questions like: "At which point and when was the highest returned intensity of the whole dataset?" or "How many clusters of points in a $0.5 \mathrm{~m}$ radius exist between 5 and 10 in $\mathrm{x}$ direction in the $124 \mathrm{~s}$ frame?". Several queries like this can be chained together to form complex pipelines. The computation is 
Geoscientific Instrumentation Methods and Data Systems

Discussions

only executed at the very last step when the answer is required by so called "lazy evaluation". The computation is performed on multiple Central Processing Units (CPUs) in parallel. The package is not limited to build-in functions and additional arbitrary functions can be implemented and applied. Furthermore, the package provides tools for visualization, import and export of widely used point cloud formats. Also a direct interface to the powerful open3D and pandas libraries (Zhou et al., 2018; The pandas development team, 2020) is implemented for additional applications. For more details see the documentation on https://virtual-vehicle.github.io/pointcloudset/.

\subsection{SLAM algorithm}

In robotics, SLAM algorithms are a fundamental prerequisite for feedback control, obstacle avoidance, and planning since SLAM allows a robot's six Degrees Of Freedom (DOF) state estimation (Bălașa et al., 2021). Here, we use a SLAM algorithm to generate one cumulative point cloud from a time-series of point clouds. MOLISENS is either mounted on a moving platform or carried along by a person while recording data. The data recording unit uses ROS as middleware and all data is recorded in a rosbag, which includes IMU, lidar, and GNSS data. Each recorded data type in the rosbag has a timestamp. The recorded data is the input for the mapping algorithm LIO-SAM (Shan et al., 2020), which is applied offline in a post-processing step.

LIO-SAM uses the lidar odometry data to estimate the six DOF trajectory of the mapping sensor. The state estimation problem is solved by a factor graph. This incorporates IMU-pre-integration, lidar odometry, GNSS data, and loop closure. The system does not depend on continuous GNSS data. Therefore, the GNSS factor is only added when the estimated position covariance is larger than the received GNSS position covariance. The loop closure factor is responsible for detecting whether a new node has a small Euclidean distance to a prior state. If this is detected, the algorithm tries to match the new state to the near, past state. This is especially useful to correct for potential drifts in altitude when GNSS is the only absolute sensor available. These advantages compared to Lidar Odometry and Mapping (LOAM) (Zhang and Singh, 2017) and other previous state of the art algorithms made it well suited for our use cases.

\section{Applications in geoscience}

250 To test the MOLISENS setup in challenging field conditions, two mapping surveys in the Lurgrotte cave system in Austria and in a glacier cave in Longyearbreen on Svalbard have been conducted. The following Section presents the results of these two measurement campaigns.

\subsection{Application in speleology}

The Lurgrotte, a partially water-bearing cave $15 \mathrm{~km}$ north of Graz in Styria, Austria, was chosen as a study area for MOLISENS. The approximately $6 \mathrm{~km}$ long cave passes through the Tanneben massif between the localities of Semriach and Peggau. Parts of the cave are accessible to tourists. The cave is characterized by an abundance of speleothems, water-bearing passages, and a heterogeneous cave geometry in which narrow passages alternate with large chambers, such as the Great Dome. With an area of approximately $5,100 \mathrm{~m}^{2}$, the Great Dome is one of the ten largest cave chambers in Austria (Plan and Oberender, 2016). 
https://doi.org/10.5194/gi-2022-3

Preprint. Discussion started: 22 February 2022

(c) Author(s) 2022. CC BY 4.0 License.

Geoscientific Instrumentation Methods and Data Systems (c) (i)

Discussions

Due to these heterogeneous characteristics, the Lurgrotte Semriach is well suited for testing the application of MOLISENS in speleology.
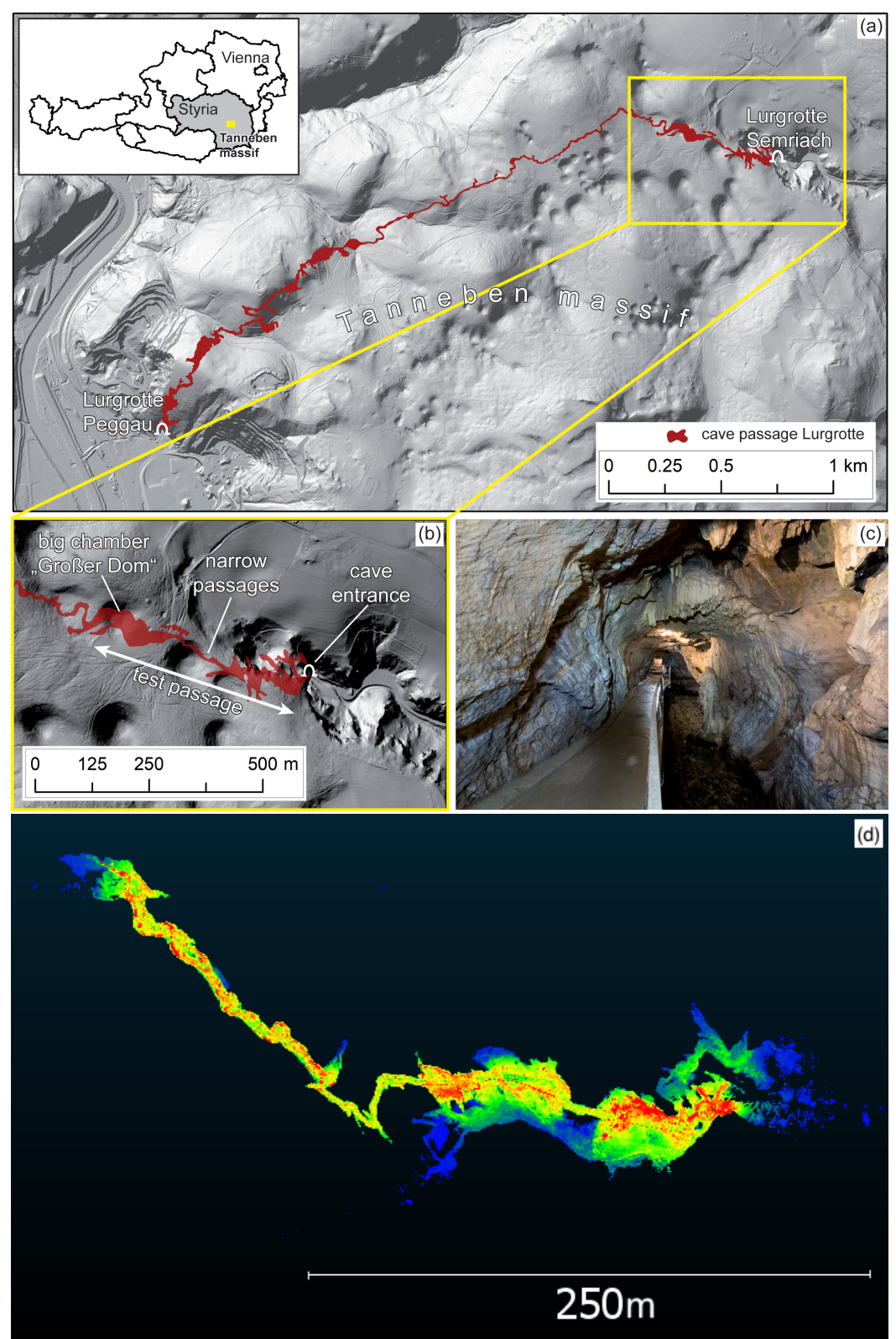

Figure 5. Study area Lurgrotte Semriach. (a) Hillshade visualization (1m $1 \mathrm{~m}$ raster resolution) and cave map (b) detailed view of the test passage in which the OS1-64 surveys were carried out including the Great Dome (Großer Dom) (c) a narrow passage with paved footpath within the cave segment open for visitors (image by Christian Bauer). Data: cave map: Bock and Dolischka (1953); ALS-data: CC-BY-4.0: Land Steiermark - data.steiermark.gv.at. (d) Measurement (2), starting at cave entrance, turning point right before the Great Dome, and end at cave entrance; point cloud colored by z-coordinate in metres, visualized with CloudCompare. 
Geoscientific Instrumentation Methods and

Data Systems

Discussions

Scanning a cave system, such as the Lurgrotte Semriach, with a TLS would demand several 10s to 100s scan positions, i.e. a significant effort in terms of time and costs. Using MOLISENS, we were able to produce a point cloud without the necessity of time consuming scans at individual positions. The mapping campaign demonstrated that MOLISENS can provide a cumulative point cloud even without the use of GNSS measurements. Also, the LIO-SAM algorithm was tested on whether it is able to co-register point clouds that were recorded partly outdoor and indoor. More than $300 \mathrm{~m}$ of complex cave geometry could be scanned with MOLISENS in less than 12 minutes (Figure 5 (d)). Measurement (1) includes the switch from an outdoor environment to an indoor environment in a single measurement. Measurement (2) was conducted only inside the cave. An overview of the recorded data is given in Table 2.

Table 2. Comparison of measurements conducted in Lurgrotte; $0.1 \mathrm{~m}$ is the minimum average point spacing that can be produced by LIOSAM

\begin{tabular}{|l|c|c|}
\hline Parameter & Measurement (1) & Measurement (2) \\
\hline \hline Duration & $6 \mathrm{~min} 37 \mathrm{~s}$ & $11 \mathrm{~min} 58 \mathrm{~s}$ \\
\hline IMU messages & 158,816 & 287,525 \\
\hline Lidar packet messages & 499,368 & 912,505 \\
\hline Size of .bag file & $6.0 \mathrm{~GB}$ & $10.9 \mathrm{~GB}$ \\
\hline Number of points in final point cloud & $10,719,001$ & $6,393,490$ \\
\hline Average point spacing & $0.1 \mathrm{~m}$ & $0.1 \mathrm{~m}$ \\
\hline
\end{tabular}

It has to be noted that a final validation of the point cloud data was not possible at this stage. A validation of the data quality and accuracy requires a geodetic reference. A marked closed traverse and local reference points were measured from the cave entrance to the Great Dome after our fieldwork at the Lurgrotte. The drilled mountings of these marks could be used used again for further tests with our system. A valid assessment of the accuracy of the produced map can then be accomplished with this reference. 


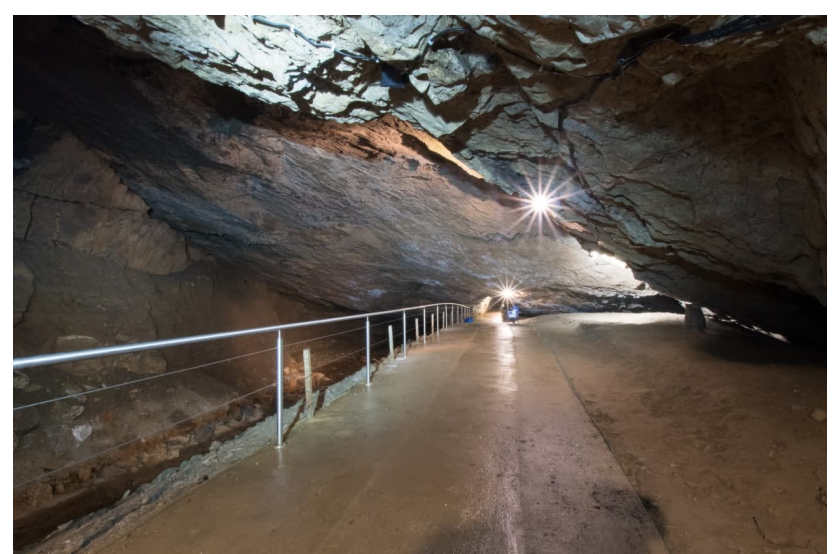

(a)

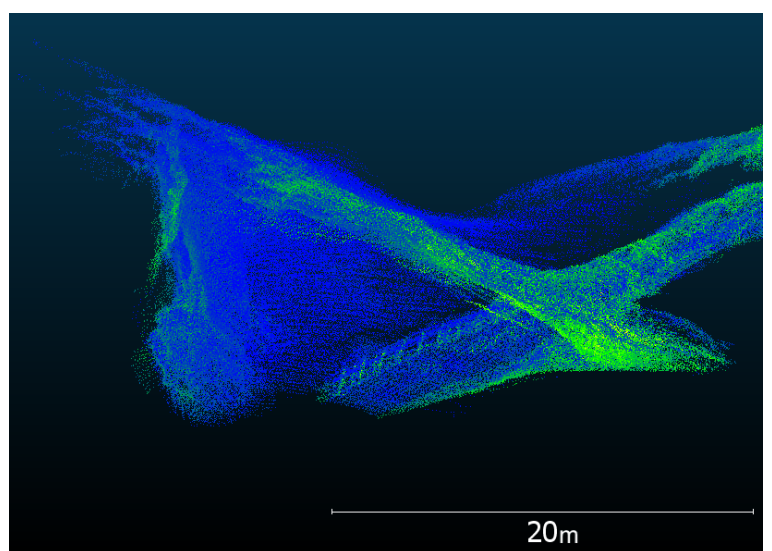

(b)

Figure 6. (a) Karst shapes, artificial concrete, and metal elements in the cave (image by Christian Bauer) (b) A detailed view on the recorded 3D pointcloud of the depicted cave section, the scanned handrails mark the walking path through the cave (visualized in CloudCompare).

\subsection{Application in glaciology}

To test MOLISENS for cryospheric applications, a glacier cave was mapped in the glacier Longyearbreen on the Svalbard archipelago, Norway. The morphological changes of glacier caves give information about the englacial water routing. Ice volume changes in caves are common throughout the year and the inter-seasonal comparison of ice dynamics can indicate a change in the hydro-climatic regime of the glacier (Perşoiu and Lauritzen, 2017). Previous work on cold glacier caves in the study area involved geomorphological mapping and seasonal temperature monitoring (Alexander et al., 2020; Guðmundsdóttir, 2011), but detailed 3D measurements of a glacier cave system are typically not available.

Our aim was to create a 3D point cloud which represent the shape of the glacier cave and the surrounding surface of the glacier. The results are shown in Figure 7. The measurement campaign showed that it is possible to create a cumulative point cloud from the predominant surfaces in and around glacier caves. These surfaces are composed of ice, snow, sediments, and moraine material. Measurements were recorded by walking through the caves bidirectionally with MOLISENS. The recorded data resulting were then processed with the LIO-SAM algorithm to create a cumulative point cloud of the glacier cave and the surrounding surface of the glacier.

Figure 7 (a) represents a segment of the processed point cloud showing the glacier cave and the glacier surface from below. The cross sections shown in Figure 7 (c) and (d) are one meter long segments along the cave direction. Some outlier points are visible that are a result of the scanners range errors, multi-path errors created by ice surfaces, and the torso of the person holding the scanner. 


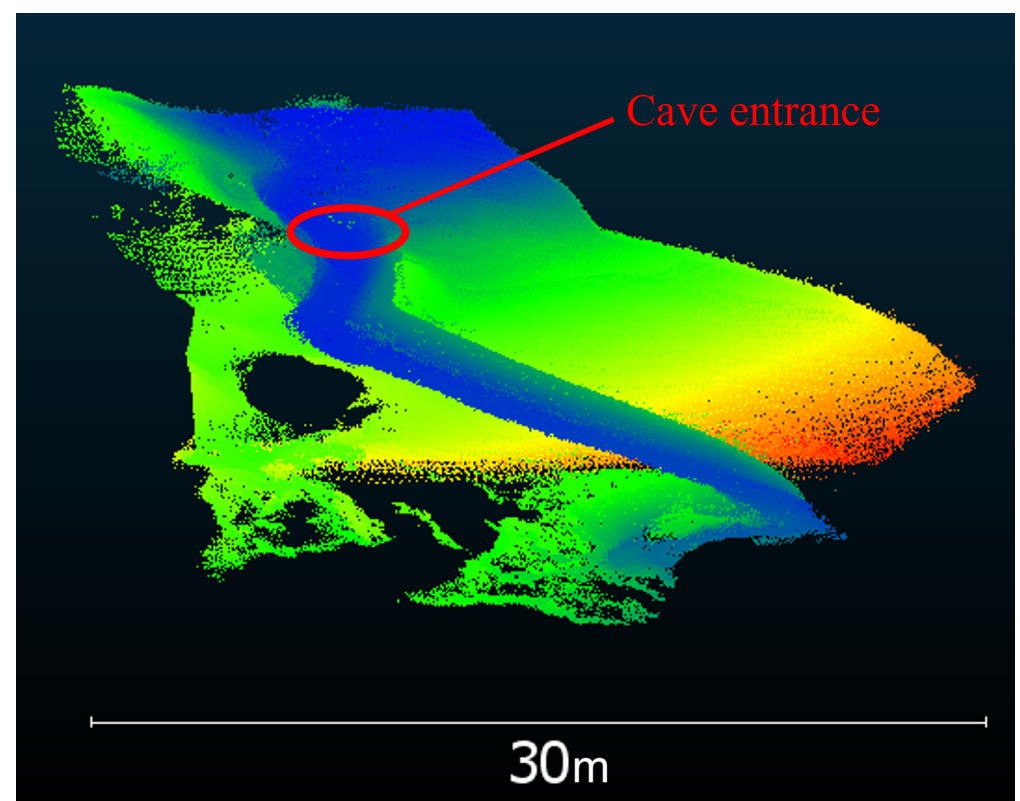

(a)

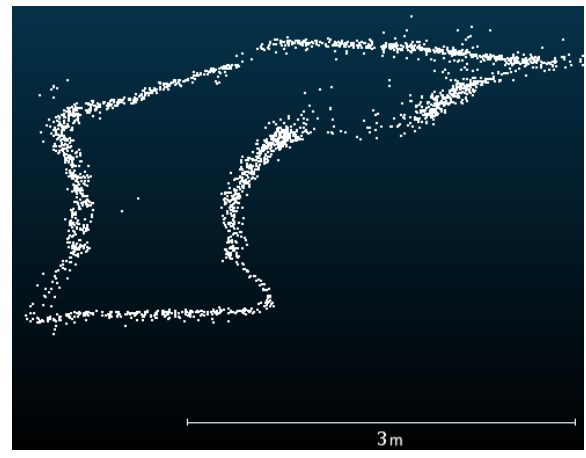

(c)

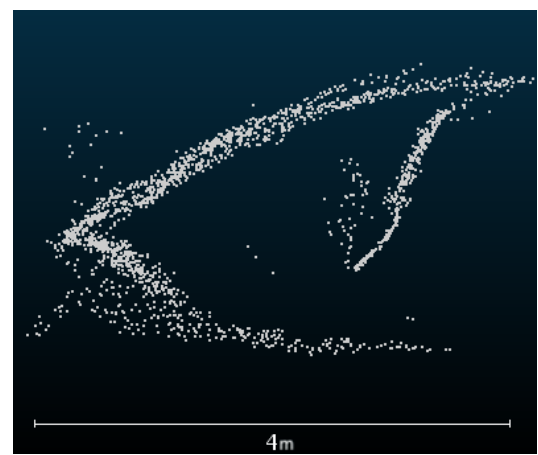

(d)

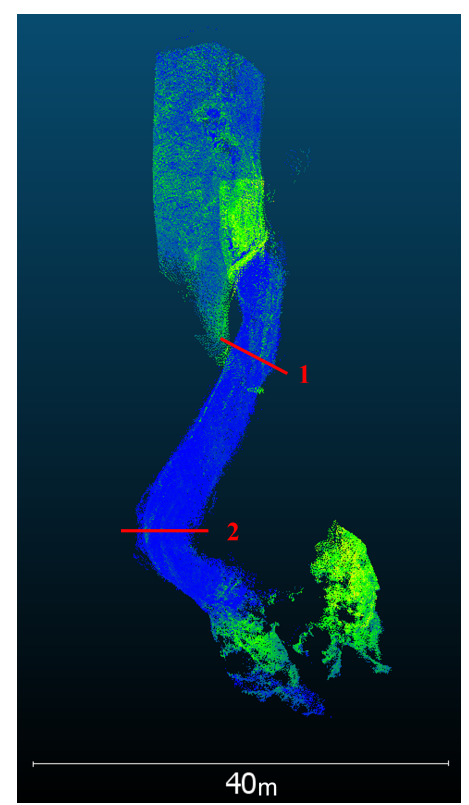

(b)

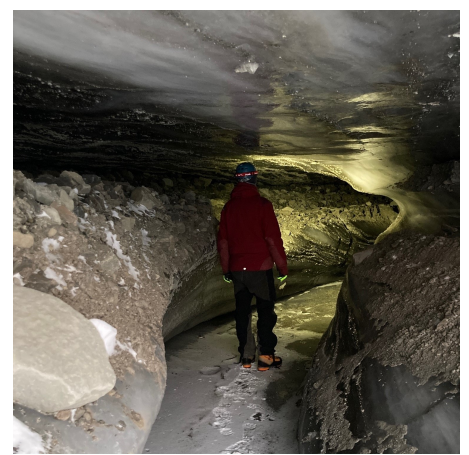

(e)

Figure 7. Resulting point cloud visualizations of glacier cave 1 on Longyearbreen (a) shows the segment of the point cloud with the glacier cave and the glacier surface (view from below) (b) shows the extracted cave (nadir view) (c) cross section 1 (d) cross section 2 (e) photo of the entrance of the cave

\section{Discussion and outlook}

\subsection{Automotive lidar as part of MOLISENS}

Our comparison shows that automotive lidar sensors such as the OS1-64 can offer advantages for certain applications compared to conventional TLS such as the VZ-6000. These are the price, size, weight, and robustness but also the ability to acquire data in narrow spaces. The accuracy of the data depends on the target properties. A black target had errors of up to $5 \mathrm{~cm}$, while a retroreflective material up to $22 \mathrm{~cm}$. Nevertheless, for numerous applications the data can be the foundation for many 
Geoscientific Instrumentation Methods and Data Systems

Discussions

kinds of analysis. We recommend to refrain from analyzing features smaller than $10 \mathrm{~cm}$ in the processed point clouds. The given accuracy of up to $5 \mathrm{~cm}$ for ranges greater than $50 \mathrm{~m}$ leads to an increase in noise when the SLAM algorithm is used. Applying smoothing filters on the cumulative point cloud is recommended. The frequency and magnitude of the distance error when scanning retroreflective surfaces is expected to be reduced with upcoming firmware version. Although, this must be considered if retroreflective targets are used during geo-referencing. Better yet would be to use non-retroreflective target for geo-referencing, e.g. white paper with black markings. The drifts in the SLAM processed point clouds has yet be quantified and workflows for geo referencing have to be tested.

In our fieldwork, MOLISENS has proven to record data in complex environments even without a GNSS signal. With the data from our fieldwork, the mapping algorithm LIO-SAM was able to map the environment and the trajectory of the mapping sensor into a point cloud. This shows that results are possible under the following conditions:

- snow and ice surfaces

- arctic weather conditions $\left(-20^{\circ} \mathrm{C}\right)$

- very narrow spaces $(<1 \mathrm{~m})$

- rough sensor handling due to rough terrain or narrow spaces

The lack of GNSS data for cave measurements caused drifts induced by small propagating errors in the IMU data. These drifts are yet to be quantified.

\subsection{Other automotive sensors}

In addition to lidar, other automotive perception sensors such as radar systems can be integrated into MOLISENS. Modern automotive and traffic monitoring radar sensors typically operate at $24 \mathrm{GHz}$, e.g., Smartmicro TRUGRD Stream (s.m.s, smart microwave sensors $\mathrm{GmbH}, 2021$ ), or $77 \mathrm{GHz}$ (Ramasubramanian and Ramaiah, 2018), e.g., Continental ARS540 (Continental AG, 2016, 2017), have a range up to 300m, and apply Frequency-Modulated Continuous Wave (FMCW) technologies for relative distance and velocity estimation (Patole et al., 2017) and digital beam-forming to control the direction of the emitted wave (Hasch, 2015). In addition to data on object level (i.e., list of detected traffic participants), radar data is typically also provided as radar clusters. Clusters represent radar detections with information like position, velocity, and signal strength. This raw data format allows to develop and apply new algorithms for detecting changes in the backscatter behavior of the environment caused by various geoscientific processes.

\subsection{Potential applications}

We envision MOLISENS as an useful tool in geosciences. The IP level of the OS1-64 allows us to conduct measurements under adverse conditions and rough sensor handling. This opens a wide range of applications ranging from cave mapping, glacier surface analysis to meltwater channel monitoring with the potential to increase our understanding of the drainage systems of 
glaciers. A 3D model of a glacier cave can be used to parameterize volumetric properties of the cave with the aim of analyzing cave morphology (Gallay et al., 2015; Šupinský et al., 2019). The recorded intensity values for ice surfaces are significantly lower than for surfaces covered with e.g. moraine material or sediments. Hence, the generated data is also a useful basis for change detection of, e.g., the ice surface (Milius and Petters, 2012).

The portable nature, low cost, and robustness of MOLISENS opens up for new applications well beyond cave mapping. Mobile high resolution 3D mapping of glacier fronts using snowmobiles on sea ice is another possible application and could be conducted at relatively high velocities (up to $60-80 \mathrm{~km} / \mathrm{h}$ ). Similarly, regular mapping of coastal bluffs susceptible to coastal erosion (e.g. Guégan and Christiansen, 2017), can be undertaken throughout the polar night season that hinder structure-frommotion photogrammetry for large parts of the year in polar regions.

Besides mobile measurements, static measurements can be conducted with MOLISENS to record rapid processes in 3D over time with up to $20 \mathrm{~Hz}$. The scanner can be placed permanently in an area of interest and in case of an event, the scanning process could be initiated automatically or remotely. Vice versa constant scanning could detect a process happening which would trigger further process chains.

MOLISENS is also a handy teaching tool since it rapidly acquires data at a fraction of the cost of a conventional TLS. In addition, it can be taken along on excursions more easily, and safety concerns are minimal even in large groups due to the laser class 1 rating. Both at the University of Graz and the University Centre in Svalbard (UNIS) it is planned to use MOLISENS for excursions and practicals focusing on cryospheric topics, mapping methods, integrated geological methods, and digital geological techniques (Senger et al., 2021).

Other potential use cases in physical geography are:

- Underground measurements: cave/mine mapping

- Monitoring glacier caves, calving glaciers

- Monitoring of snow and avalanches

- Monitoring of mass movements

- Monitoring of fluvial systems

- Monitoring of erosion and deposition processes

- Sea ice detection and mapping

- Coastal mapping

- Archaeology, historical and cultural preservation

- Forestry surveys

Potential use cases in urban environments are: 
https://doi.org/10.5194/gi-2022-3

Preprint. Discussion started: 22 February 2022

(c) Author(s) 2022. CC BY 4.0 License.

(c) (i)

Geoscientific Instrumentation

Methods and

Data Systems

Discussions

- Highways surveys

- Roadside inventory projects

- Power line corridor surveys

- 3D City modeling

- 3D indoor modeling

This list can be further extended since the system can be attached to a wide range of platforms. Tests have been conducted with platforms like cars, agricultural machines, and boats with promising results. Further optimizing weight and power consumption of the system can also enable small Unmanned Aerial Vehicles (UAVs)s as potential platforms. Apart from geoscientific applications, MOLISENS provides an easy to use setup for testing automotive perception sensors for e.g. sensor modeling and sensor Fault Detection, Identification, and Recovery (FDIR) method development.

\section{Conclusion}

In this work, we present a newly developed mobile lidar sensor system called MOLISENS. The system combines an automotive lidar with IMU and GNSS. It provides the opportunity to collect 3D data for a wide range of use cases and applications. Besides the hardware we introduced the post-processing tools provided by the two open source packages LIO-SAM and pointcloudset. LIO-SAM is a SLAM algorithm for cumulative point cloud generation, and pointcloudset a Python package for analysis and post-processing of static measurements.

The integration of the automotive lidar OS1-64 and the mobile mapping approach was tested in measurement campaigns in the Lurgrotte cave, Austria and in glacier caves on Longyeabreen, Svalbard. The system offers a flexible, easy to use, and time-efficient way to acquire 3D point cloud, GNSS, and IMU data. The offline SLAM processing resulted in point clouds which can be the basis to investigate numerous geoscientific problems. The robustness of the sensors and the data logger as well as the battery and storage capacities are well suited to demanding fieldwork situations.

In the near future, additional sensors, such as radar and camera, shall be integrated into MOLISENS and further broaden the range of applications. This is possible due to the modular design structure of MOLISENS.

Code and data availability. pointcloudset is available at https://github.com/virtual-vehicle/pointcloudset and LIO-SAM at https://github. com/TixiaoShan/LIO-SAM

Sample availability. Point could data from Longyearbreen Glacier Cave, Svalbard is available at https://doi.org/10.3217/182j2-hdn17 
https://doi.org/10.5194/gi-2022-3

Preprint. Discussion started: 22 February 2022

(C) Author(s) 2022. CC BY 4.0 License.

(c) (i)

Geoscientific
Instrumentation
Methods and
Data Systems
Discussions

Competing interests. No competing interests are present.

Acknowledgements. The publication was written at the University of Graz and at the Virtual Vehicle Research GmbH. The authors would

385 like to acknowledge the financial support within the COMET K2 Competence Centers for Excellent Technologies from the Austrian Federal Ministry for Climate Action (BMK), the Austrian Federal Ministry for Digital and Economic Affairs (BMDW), the Province of Styria (Dept. 12) and the Styrian Business Promotion Agency (SFG). The Austrian Research Promotion Agency (FFG) has been authorised for the programme management. A special thanks to Oliver Mariani from Virtual Vehicle Research GmbH for supporting the construction of several hardware parts of MOLISENS. The authors would like to acknowledge Andreas Schinnerl for providing access to the Lurgrotte Semriach and supporting the measurements. The fieldwork in Svalbard was in part funded by the Svalbard Science Forum Arctic Field Grant. The authors acknowledge the financial support by the University of Graz. 
Geoscientific Instrumentation Methods and Data Systems

Discussions

\section{References}

AccuPower Research, Development and Distribution Company (Ltd.): AccuPower AkkuPacks, https://www.accupower.at/produkt-kategorie/ akkus/lithium/akkupacks/, accessed 01 February 2022, 2022.

Alexander, A., Obu, J., Schuler, T. V., Kaab, A., and Christiansen, H. H.: Subglacial permafrost dynamics and erosion inside subglacial channels driven by surface events in Svalbard, The Cryosphere, 14, 4217-4231, https://doi.org/10.5194/tc-14-4217-2020, 2020.

Bălașa, R. I., Olaru, G., Constantin, D., Ștefan, A., Bîlu, C. M., and Bălăceanu, M. B.: LIDAR based distance estimation for emergency use terrestrial autonomous robot, 14th International Conference on Electronics, Computers and Artificial Intelligence (ECAI), 13, 1-4, https://doi.org/10.1109/ECAI52376.2021.9515047, 2021.

Behley, J. and Stachniss, C.: Efficient Surfel-Based SLAM using 3D Laser Range Data in Urban Environments, in: Conference: Robotics: Science and Systems 2018, Pittsburgh, Pennsylvania, USA, https://doi.org/10.15607/RSS.2018.XIV.016, 2018.

Birkebak, M., Stearns, J., Durell, C., and Scharpf, D.: Radiometry 101 Calibrating with diffuse reflecting targets, https://www. photonicsonline.com/doc/radiometry-calibrating-with-diffuse-reflecting-targets-0001, 2018.

Bock, H. and Dolischka, A.: Plan der Lurgrotte Peggau - Semriach, Tech. rep., Graz, m = 1:2.500, 1953.

Boehler, W., Vicent, M., and Marbs, A.: Investigating laser scanner accuracy, in: Proc. CIPA XIXth Int, Symposium. $34,2003$.

Bosse, M., Zlot, R., and Flick, P.: Zebedee: Design of a Spring-Mounted 3-D Range Sensor with Application to Mobile Mapping, IEEE Transactions on Robotics, 28, 1104-1119, https://doi.org/10.1109/TRO.2012.2200990, 2012.

Continental AG: Technical Documentation ARS 404-21 (Entry) and ARS 408-21 (Premium), Version 1.0, Tech. rep., Continental Engineering Services GmbH, 2016.

Continental AG: ARS 408-21 Premium Long Range Radar Sensor 77 GHz, ARS 408-21 datasheet, Tech. rep., Continental Engineering Services $\mathrm{GmbH}, 2017$.

Druml, N., Maksymova, I., Thurner, T., van Lierop, D., Hennecke, M., and A., F.: 1d Mems Micro-Scanning LiDAR, Conference on Sensor Device Technologies and Applications (SENSORDEVICES), 9, 2018.

Dunning, D.: What Is Difference Between RTK Fix and RTK Float?, https://sciencing.com/difference-between-rtk-fix-rtk-float-12245568. html, accessed 04 February 2022, 2018.

Gallay, M., Kaňuk, J., Hochmuth, Z., Meneely, J., Hofierka, J., and Sedlák, V.: Large-scale and high-resolution 3-d cave mapping by terrestrial laser scanning: A case study of the Domica cave, Slovakia, International Journal of Speleology, 44, 277-291, 2015.

Goelles, T., Schlager, B., Muckenhuber, S., Haas, S., and Hammer, T.: pointcloudset: Efficient Analysis of Large Datasets of Point Clouds Recorded Over Time, Journal of Open Source Software, 6, 3471, https://doi.org/10.21105/joss.03471, 2021.

Guégan, E. B. M. and Christiansen, H. H.: Seasonal Arctic Coastal Bluff Dynamics in Adventfjorden, Svalbard, Permafrost and Periglac. Process, 28, 18-31, https://doi.org/10.1002/ppp.1891, 2017.

Guðmundsdóttir, A. S.: Morphology and Development of the Longyearbreen Ice Cave, Central Spitsbergen, Svalbard, Bachelor Thesis BS, ritgerð, Jarðvísin-dadeild, Háskóli Íslands, 2011.

Hammer, T.: New applications of automotive lidar sensors in geosciences, mathesis, TU Graz, 2021.

Hasch, J.: Driving Towards Automotive Radar Technology Trends, IEEE MTT-S International Conference on Microwaves for Intelligent Mobility, pp. 1-4, 2015.

Hecht, J.: Lidar for self-driving cars, Optics and Photonics News, 29, 26-33, 2018. 
https://doi.org/10.5194/gi-2022-3

Preprint. Discussion started: 22 February 2022

(c) Author(s) 2022. CC BY 4.0 License.

Geoscientific Instrumentation Methods and Data Systems

Discussions

Ibeo Automotive Systems GmbH: ibeo LUX 4L / ibeo LUX 8L / ibeo LUX HD Datasheet, https://hexagondownloads.blob.core.windows. net/public/AutonomouStuff/wp-content/uploads/2019/05/ibeo_LUX_datasheet_whitelabel.pdf, accessed 4 October $2021,2021$.

Janos, D. and Przemysław, K.: Evaluation of Low-Cost GNSS Receiver under Demanding Conditions in RTK Network Mode, Sensors 21, 16, 5552, https://doi.org/10.3390/s21165552, 2021.

Kukko, A., Kaartinen, H., Hyyppä, J., and Chen, Y.: Multiplatform mobile laser scanning: Usability and performance, Sensors, 12, 11 712$11733,2012$.

Leica Geosystems AG: Leica ScanStation P30/P40: Leica P30/P40 data sheet, https://leica-geosystems.com/-/media/files/leicageosystems/ products/datasheets/scan/leica\%20scanstation\%20p30-p40\%20ds\%20832252\%200119\%20en\%20arch\%20bui\%20bim\%20lr.ashx?la= de-at\&hash=FE9B9FD3A500B5D6332AF0439D725BD8, accessed 4 October 2021, 2021a.

Leica Geosystems AG: Leica ScanStation P50: Leica P50 data sheet, https://leica-geosystems.com/-/media/files/ leicageosystems/products/datasheets/scan/leica\%20scanstation $\% 20 \mathrm{p} 50 \% 20 \mathrm{ds} \% 20869145 \% 200119 \% 20 \mathrm{en} \% 201 \mathrm{l}$.ashx? $\mathrm{la}=\mathrm{de}-\mathrm{at} \&$ hash= 9ABF78CC529268400306349359BE769A, accessed 4 October 2021, $2021 \mathrm{~b}$.

440 Marti, E., Perez, J., Miguel, M. A., and Garcia, F.: A Review of Sensor Technologies for Perception in Automated Driving, IEEE Intelligent Transportation Systems Magazine, pp. 94-108, https://doi.org/10.1109/MITS.2019.2907630, 2019.

Milius, J. and Petters, C.: Eisriesenwelt - from laser scanning to photo-realistic 3d model of the biggest ice cave on earth, GI-Forum 2012: Geovisualization,Society and Learning, Salzburg, 513, 513-523, 2012.

Moosmann, F. and Stiller, C.: Velodyne SLAM, in: Proceedings of the IEEE Intelligent Vehicles Symposium, pp. 393-398, Baden-Baden, Germany, http://www.mrt.kit.edu/z/publ/download/Moosmann_IV11.pdf, 2011.

Muckenhuber, S., Holzer, H., and Bockaj, Z.: Automotive lidar modelling approach based on material properties and lidar capabilities, Sensors (Switzerland), 20, 1-26, 2020.

Nahler, C., Steger, C., and Druml, N.: Quantitative and qualitative evaluation methods of automotive time of flight based sensors, in: A., editors, Proceedings - Euromicro Conference on Digital System Design, DSD 2020, edited by Trost, A., Zemva, A., and Skavhaug, A., pp. 651-659, Institute of Electrical and Electronics Engineers, United States., 2020.

Ouster Inc.: Ouster Example Code, https://github.com/ouster-lidar/ouster_example/tree/20201209, accessed 01 February 2022, 2020a.

Ouster Inc.: OS1 Mid-Range High resolution Imaging Lidar, Ouster OS-1 Gen1 data sheet, https://data.ouster.io/downloads/datasheets/ datasheet-gen1-v2p0-os1.pdf, accessed 13 December 2021, 2020 b.

Ouster Inc.: OSO Ultra-Wide View High-Resolution Imaging Lidar, Ouster OS-0 data sheet, https://data.ouster.io/downloads/datasheets/ datasheet-revd-v2p1-os0.pdf, accessed 4 October 2021, 2021a.

Ouster Inc.: OS1 Mid-Range High resolution Imaging Lidar, Ouster OS-1 data sheet, https://data.ouster.io/downloads/datasheets/ datasheet-revd-v2p1-os1.pdf, accessed 4 October 2021, 2021 b.

Ouster Inc.: OS2 Long-Range High-Resolution Imaging Lidar, Ouster OS-2 data sheet, https://data.ouster.io/downloads/datasheets/ datasheet-revd-v2p1-os2.pdf, accessed 4 October 2021, 2021c.

460 Ouster Inc.: ES2 Solid-State Lidar is now Digital, https://ouster.com/products/es2-solid-state-lidar-sensor/, accessed 12 January $2022,2022$.

Patole, S. M., Torlak, M., Wang, D., and Murtaza, A.: Automotive radars: A review of signal processing techniques, IEEE Signal Processing Magazine, 34, 22-35, https://doi.org/10.1109/MSP.2016.2628914, 2017.

Perşoiu, A. and Lauritzen, S.-E.: Ice Caves, Elsevier, https://doi.org/https://doi.org/10.1016/C2016-0-01961-7, 2017.

Plan, L. and Oberender, P.: Höhlen und Karst in Österreich, chap. Höhlen in Österreich, pp. 11-22, Oberösterreichisches Landesmuseum, 2016. 
https://doi.org/10.5194/gi-2022-3

Preprint. Discussion started: 22 February 2022

(c) Author(s) 2022. CC BY 4.0 License.

(c) (i)

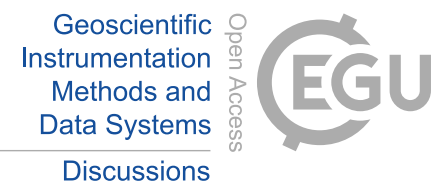

Ramasubramanian, K. and Ramaiah, K.: Moving from Legacy 24 GHz to State-of-the-Art 77-GHz Radar, ATZelektronik worldwide, 13, 46-49, https://doi.org/10.1007/s38314-018-0029-6, 2018.

Rieg1 Laser Measurement Systems GmbH: 3D Ultra Long Range Terrestrial Laser Scanner with Online Waveform Processing Riegl VZ-6000, http://www.riegl.com/uploads/tx_pxpriegldownloads/RIEGL_VZ-6000_Datasheet_2020-09-14.pdf, accessed 4 October 2021, 2020.

Senger, K., Betlem, P., Grundvag, S. A., Horota, R. K., Buckley, S. J., Smyrak-Sikora, A., Jochmann, M. M., Birchall, T., Janocha, J., Ogata, K., Kuckero, L., Johannessen, R. M., Lecomte, I., Cohen, S. M., and Olaussen, S.: Teaching with digital geology in the high Arctic: opportunities and challenges, Geoscience Communication, 4, 399-420, https://doi.org/10.5194/gc-4-399-2021, 2021.

Shan, T., Englot, B., Meyers, D., Wang, W., Ratti, C., and Daniela, R.: Lio-sam: Tightly-coupled lidar inertial odometry via smoothing and mapping, IEEE/RSJ International Conference on Intelligent Robots and Systems (IROS), pp. 5135-5142, 2020.

s.m.s, smart microwave sensors GmbH: SmartMicro Product information, traffic management sensor, TRUGRD Stream, https: //www.smartmicro.com/fileadmin/media/Downloads/Traffic_Radar/Sensor_Data_Sheets_24_GHz_/Datasheet_TRUGRD_Stream.pdf, accessed 4 October 2021, 2021.

Šupinský, J., Kaňuk, J., Hochmuth, Z., and Gallay, M.: Detecting dynamics of cave floor ice with selective cloud-to-cloud approach, The Cryosphere, 13, 2835-2851, 2019.

Thakur, R.: Scanning LIDAR in Advanced Driver Assistance Systems and Beyond: Building a road map for next-generation LIDAR technology, IEEE Consumer Electronics Magazine, 5, 48-54, 2016.

The pandas development team: pandas-dev/pandas: Pandas, https://doi.org/10.5281/zenodo.3509134, 2020.

Tucci, G., Visintini, D., Bonora, V., and Parisi, E. I.: Examination of Indoor Mobile Mapping Systems in a Diversified Internal/External Test Field, Applied Sciences, 8, https://doi.org/10.3390/app8030401, 2018.

Velodyne Lidar Inc.: Velodyne Lidar Alpha Prime: Velodyne Alpha Prime data sheet, https://velodynelidar.com/products/alpha-prime/, accessed 4 October 2021, 2021.

Wang, Y., Liang, X., Flener, C., Kukko, A., Kaartinen, H., Kurkela, M., Vaaja, M., Hyyppä, H., and Alho, P.: 3d modeling of coarse fluvial sediments based on mobile laser scanning data, Remote Sensing, 5, 4571- 4592, 2013.

Watzenig, D. and Horn, M.: Automated Driving - Safer and More Efficient Future Driving, Springer, Cham, https://doi.org/https://doi.org/10.1007/978-3-319-31895-0, 2017.

Xsens: Download MT Software Suite 2021.4, https://content.xsens.com/mt-software-suite-download, accessed 01 February $2022,2021$.

Zhang, J. and Singh, S.: Low-drift and Real-time Lidar Odometry and Mapping, Autonomous Robots, 41, 401-416, https://doi.org/10.1007/s10514-016-9548-2, 2017.

Zhou, Q.-Y., Park, J., and Koltun, V.: Open3D: A Modern Library for 3D Data Processing, arXiv:1801.09847, 2018. 